\title{
A Survey on Datacenter Placement and Replication Approach for Cloud
}

\author{
Prachi Shrivastava \\ (UIT) RGPV Bhopal, India
}

\author{
Uday Chourasia \\ (UIT) RGPV Bhopal, India
}

\author{
Shikha Agrawal, PhD \\ (UIT) RGPV Bhopal, India
}

\begin{abstract}
Cloud computing is a trending technology due to its high availability and services. Cloud provides a platform for the large set of users to put their data and host for their processing requirements. But the main challenge is to provide a reliable storage for the users. To provide reliability cloud uses concept of replication in datacenters. But in case of natural calamity it is critical to save at least one replica of data; which will not be possible if all replicas are made in disaster prone locations. Apart from this network usage, energy consumption and service level agreement (SLA) should also be kept in mind. In this paper a survey is done on intelligent replication techniques which will keep all performance parameters in consideration. There is SLA violation and service can be improved considerably if data placement and replication algorithms are better.
\end{abstract}

\section{Keywords}

Cloud, Datacenter, replication, Service level agreement (SLA).

\section{INTRODUCTION}

These days digitization has been rapidly increasing throughout the world. As a result of which data is also increasing. To handle this data and to analyze this data several techniques are introduced. One of such techniques is cloud computing which also handles big data. Apart from that cloud computing is mainly used to provide access to online resources which if maintained and owned by client would have been very costly. The biggest challenge of cloud computing is to provide service to users which should be reliable and available $24 \times 7$. In order to provide a reliable source of data storage to clients cloud has to use concept of fault tolerant. Cloud uses replication to provide fault tolerant architecture [2]. There are many frameworks to provide cloud management and development; Hadoop is one such framework. Replication is managed by Hadoop management service itself [3]. In this paper a disaster aware replication approach for cloud computing is studied. As if all the replicas are stored in disaster prone locations then it will be of no use. So here based on the past natural calamities data replication location is choose accordingly.

Cloud computing prime aim is to provide a reliable service to its client where client can store his data and also use virtual servers for processing. Cloud is a transition from distributed processing to online accessible cluster of machines which can be used by a number of users. This cluster of machines is managed by a cluster computing framework called Hadoop. However Hadoop is an open source parallel processing framework for big data processing but can also be used to implement cloud.

The paper is organized as follows: Section II describes cloud terminologies and architecture. Section III describes the apache Hadoop framework which is a big data analytics tool and also used as a framework to provide cloud services. In section IV previous work done in the past by researchers is discussed. Section V summarizes the techniques discussed for intelligent replication.

\section{ARCHITECTURE OF CLOUD}

Cloud is nothing but collection of resources like storage, processing elements which can be accessed online by a number of users simultaneously. It consists of three layers Infrastructure as a service (IaaS), Platform as a service (PaaS) and Software as a service (SaaS). To distribute data across various datacenters which are geographically separated is the responsibility of IaaS layer.

Table 1: Terminologies in Cloud Computing

\begin{tabular}{|l|l|}
\hline \multicolumn{2}{|c|}{ Cloud Terminology } \\
\hline Cloudlet & $\begin{array}{l}\text { Task submitted by the users } \\
\text { is termed as cloudlet in } \\
\text { cloud computing }\end{array}$ \\
\hline Datacenter & $\begin{array}{l}\text { Collection of resources } \\
\text { which includes storage and } \\
\text { server (Physical machines } \\
\text { PM) }\end{array}$ \\
\hline Cloud Information Service & $\begin{array}{l}\text { This is an agent which } \\
\text { handles resource allocation } \\
\text { from datacenter. This is not } \\
\text { exposed to user through } \\
\text { direct communication due to } \\
\text { security reasons. }\end{array}$ \\
\hline Virtual Machine (VM) & $\begin{array}{l}\text { A VM is created for every } \\
\text { cloudlet and this VM is } \\
\text { assigned a PM by Cloud } \\
\text { information service or } \\
\text { Hypervisor. }\end{array}$ \\
\hline Broker & $\begin{array}{l}\text { Broker is an agent which } \\
\text { takes the cloudlet from the } \\
\text { user and communicates with } \\
\text { the Cloud information } \\
\text { service and submits virtual } \\
\text { machine to the datacenter. }\end{array}$ \\
\hline
\end{tabular}

Cloudlet is submitted by the user to the broker. Broker requests Cloud information service to allocate physical machine. It replies with a token and PM number. Then broker directly submits VM on the specified PM. This process is illustrated in diagram below. 


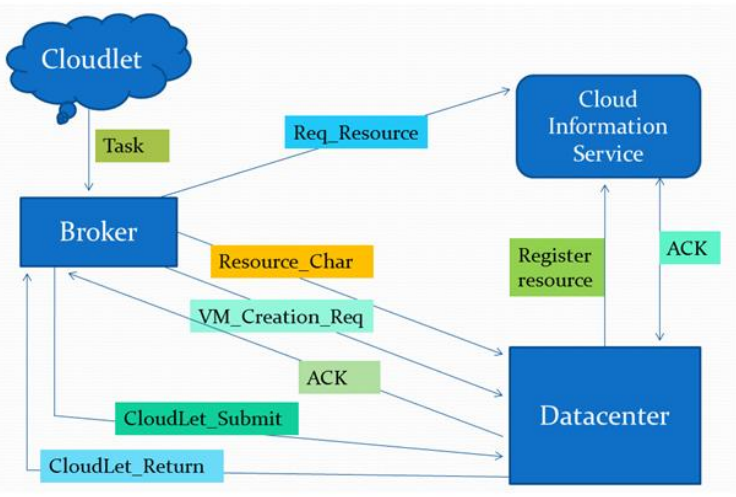

Fig 1: Architecture of Cloud

\subsection{Hadoop}

Hadoop is an apache foundation open source big data processing framework which is also used for cloud computing. Hadoop provides cluster management services which include services to put data on various datacenters distributed across different geographical locations. Hadoop has two basic components:

- Hadoop distributed filesystem (HDFS).

- Yet another resource negotiator (YARN).

The first component is of our concern as far as this survey is concerned. There are daemons running in background to provide services. HDFS has two daemons named Namenode which runs on master node of the cluster and Datanode which runs on all the worker nodes of Hadoop cluster which serves as cloud for clients. All the systems will have their storage racks which may be separated geographically.

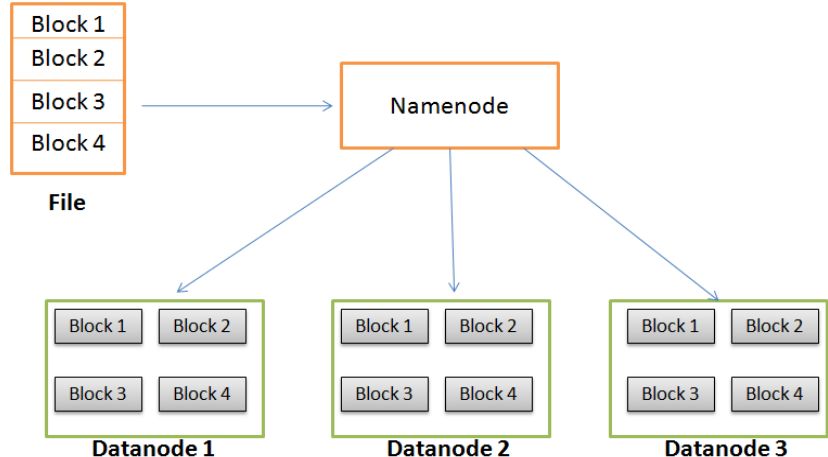

Fig 2: Replication of blocks on Hadoop

The above figure illustrates that file is divided into blocks and every block is placed by data placement algorithm and each block is replicated thrice by default. So the prime concern of this paper is to make these replications of blocks in different geographical locations such that all are not disaster prone.

\section{INTELLIGENT REPLICATION APPROACHES}

A number of researchers had proposed several techniques for intelligent disaster prone replication scheme. In addition to disaster aware replication some parameters like energy consumption, network usage and SLA are also taken in consideration. In [10] authors proposed a data placement approach for datacenters according to number of users, power availability. In [18] according to failure model data is placed. In [16] replication and data is placed according to latency i.e. it should be placed geographically closest to the users which will use it. Because with decrease in latency network usage and energy consumption of cloud will also decrease and thus SLA violation will decrease. In [12], a study is done on content or replica placement in a datacenter network with the objective of reducing network cost and latency $[5,6]$.

\section{COMPARISON BETWEEN DIFFERENT REPLICATION} APPROACHES

\begin{tabular}{|c|c|c|c|}
\hline APPROACH NAME & DESCRIPTION & DRAWBACKS & REFERENCES \\
\hline $\begin{array}{c}\text { Disaster based content } \\
\text { placement approach }\end{array}$ & $\begin{array}{c}\text { In this approach content is } \\
\text { allocated physical machines } \\
\text { in datacenter depending upon } \\
\text { the geographical location of } \\
\text { the datacenter. }\end{array}$ & $\begin{array}{c}\text { All the reliability of this } \\
\text { approach depends on } \\
\text { classification and analysis for } \\
\text { finding safe and disaster } \\
\text { prone locations. }\end{array}$ & [6] [1] \\
\hline $\begin{array}{c}\text { Intelligent replication } \\
\text { approach }\end{array}$ & $\begin{array}{c}\text { In this approach replication } \\
\text { of every block of the content } \\
\text { is done on different locations } \\
\text { such that all of those should } \\
\text { not fall in disaster prone } \\
\text { locations }\end{array}$ & $\begin{array}{c}\text { All the reliability of this } \\
\text { approach depends on } \\
\text { classification and analysis for } \\
\text { finding safe and disaster } \\
\text { prone locations. }\end{array}$ & [7] [8] \\
\hline $\begin{array}{c}\text { Machine learning for disaster } \\
\text { location clustering }\end{array}$ & $\begin{array}{c}\text { In this approach machine } \\
\text { learning is applied to cluster } \\
\text { locations in to disaster prone } \\
\text { locations and safe locations. }\end{array}$ & $\begin{array}{c}\text { Here clustering is done on the } \\
\text { basis of real time data and } \\
\text { current scenarios and } \\
\text { predications so this is better } \\
\text { but it posses considerable } \\
\text { overhead. }\end{array}$ & \\
\hline
\end{tabular}


A. Machine learning for intelligent replication on datacenters

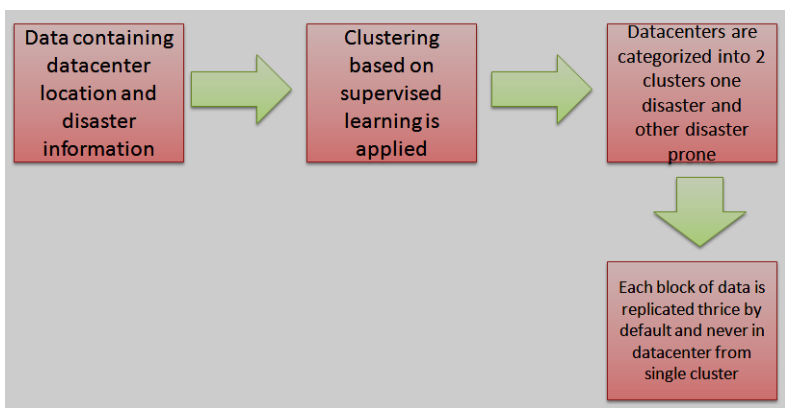

Fig 3: Figure showing overview of intelligent replication approach for cloud

\section{CONCLUSION}

In this paper a survey is done on disaster aware replication algorithms for cloud computing. Different replication approaches are studied. But the main concern is about the replication approach because these replications are formed dynamically and in a very short span of time so there has to be quick action and thus short time span algorithms are necessary which may need machine learning approaches also.

\section{REFERENCES}

[1] M. F. Habib, M. Tornatore, M. De Leenheer, F. Dikbiyik, and B. Mukherjee, "Design of disaster-resilient optical datacenter networks," J. Lightwave Technol., vol. 30, no. 16, pp. 2563-2573, Aug. 2012.

[2] C. Develder, M. De Leenheer, B. Dhoedt, M. Pickavet, D. Colle, F. D. Turck, and P. Demeester, "Optical networks for grid and cloud computing applications," Proc. IEEE, vol. 100, no. 5, pp. 1149-1167, May 2012.

[3] C. Lam, H. Liu, B. Koley, X. Zhao, V. Kamalov, and V. Gill, "Fiber optic communication technologies: What's needed for datacenter network operations," IEEE Commun. Mag., vol. 48, no. 7, pp. 32-39, July 2010.

[4] http://www.datacenterknowledge.com/archives/2013/01/15/ amazon-to-add-capacity-to- ${ }^{-}{ }^{-}$- $^{-}$- east-region/.

[5] http://www.seattletimes.com/seattle-news/alaska-airlinesourticketing-system-up-and-running-again/.

[6] http://www.techradar.com/us/news/world- of tech/roundup/onein-two-businesses-will-experience -data-loss-this-year-1093376.

[7] B. Mukherjee, M. F. Habib, and F. Dikbiyik, "Network adaptability from disaster disruptions and cascading failures," IEEE Commun. Mag., vol. 52, no. 5, pp. 230238, May 2014.

[8] A. Bernstein, D. Bienstock, D. Hay, M. Uzunoglu, and G. Zussman, "Power grid vulnerability to geographically correlated failures - analysis and control implications," Columbia University, Tech. Rep., June 2012.

[9] F. Dikbiyik, M. Tornatore, and B. Mukherjee, "Minimizing the risk from disaster failures in optical backbone networks," J. Lightwave Technol., vol. 32, no. 18, pp 3175-3183, 2014.

[10] http://www.google.com/about/datacenters/inside/locations/.

[11] http://finance.yahoo.com/news/yahoos-data-center-ny-194 329808 .html.

[12] A. Greenberg, J. Hamilton, D. Maltz, and P. Patel, "The cost of a cloud: Research problems in data center networks," ACM SIGCOMM Comput. Commun. Rev., vol. 39, no. 1, pp. 68-73, Jan. 2009.

[13] J. Xiao, B. Wu, X. Jiang, P. Ho, and S. Fu, "Data center network placement and service protection in all-optical mesh networks," in Proc. DRCN, Hungary, Mar. 2013.

[14] X. Dong, T. El-Gorashi, and J. Elmirghani, "Green IP over WDM networks with data centers," J. Lightwave Technol., vol. 29, no. 12, pp. 1861-1880, June 2011.

[15] Y. Chen, R. H. Katz, and J. D. Kubiatowicz, "Dynamic replica placement for scalable content delivery," in Proc. Int.Workshop on Peer-To-Peer (IPTPS), Cambridge, MA, Mar. 2002.

[16] N. Bartolini, F. Presti, and C. Petrioli, "Optimal dynamic replica placement in content delivery networks," in Proc. Int. Conf. on Networks (ICON), Sydney, Australia, Sept. 2003, pp. 125-130.

[17] P. Agarwal, A. Efrat, S. Ganjugunte, D. Hay, S.Sankararaman, and G. Zussman, "The resilience of WDM networks to probabilistic geographical failures," in Proc. IEEE Int. Conf. on Computer Communications (INFOCOM), Shanghai, China, Apr. 2011, pp. 1521-1529.

[18] X. Wang, X. Jiang, and A. Pattavina, "Assessing network vulnerability under probabilistic region failure model," in Proc. IEEE High Performance Switching and Routing Conf. (HPSR), Cartagena, Spain, July 2011.

[19] http://googleenterprise.blogspot.com/2010/03/disaster-reco veryby-google. html.

[20] http://en.wikipedia.org/wiki/Recovery_point_objective.

[21] http://wikibon.org/wiki/v/Defining_RPO_and_RTO.

[22] L. Jiao, J. Li, T. Xu, and X. Fu, "Cost optimization for online social networks on geo-distributed clouds," in Proc. Int. Conf. on Network Protocols (ICNP), 2012, pp. 1-10.

[23] https://developers.google.com/storage/pricing\#_PricingExa mple.

[24] http://azure.microsoft.com/enus/pricing/overview/.

[25] https://www.synaptic.att.com/clouduser/.

[26] http://aws.amazon.com/s3/pricing.

[27] ]http://searchstorage.techtarget.co.uk/tip/Cloudstorage-prici ngrevealed-Hidden-costs-include-data-migration-and-ac cessfees.

[28] T. L. Weems, "How far is far enough," Disaster Recov. J., vol. 16 , no. 2,2003 